\title{
ORIGINAL
}

\section{OPINIÓN Y PARTICIPACIÓN DE LOS MÉDICOS DE FAMILIA DE LA COMUNIDAD DE MADRID EN EL PROGRAMA REGIONAL DE DETECCIÓN PRECOZ DE CÁNCER DE MAMA EN 2007}

\author{
Sofía Garrido Elustondo (1), Elisabeth Sánchez Padilla (2), Victoria Ramírez Alesón (1), Ma José \\ González Hernández (3), Andrés González Navarro (3) y Carlos López Gómez (1) \\ (1) Gerencia de Atención Primaria. Area 7. Madrid. \\ (2) Servicio de Medicina Preventiva. Hospital Clínico San Carlos. Madrid. \\ (3) Oficina Regional de Coordinación Oncológica Servicio Madrileño de Salud.
}

\section{RESUMEN}

Fundamento: La mamografía de cribado es el método más efectivo para la detección precoz de cáncer de mama. El objetivo de nuestro estudio es valorar el grado de conocimiento, opinión y participación de los médicos de familia de la Comunidad de Madrid en el programa de detección precoz de cáncer de mama.

Métodos: La población de estudio fueron los médicos de familia del Area 7 de Madrid. Se diseñó un cuestionario anónimo autoadministrado, con 30 preguntas, agrupadas en características del médico, y opinión sobre los programas de detección precoz de cáncer de mama.

Resultados: Se obtuvo un respuesta del 46\%. El 94\% de los médicos creen que es función suya informar a sus pacientes acerca de las actividades preventivas, incluidas el cribado de cáncer de mama, y el $95 \%$ creen que su consejo es útil para convencer a las mujeres a que se realicen una mamografía. El $72 \%$ cree que le falta información sobre el programa. Durante el periodo de realización de mamografías en su centro, el $24 \%$ pregunta siempre o casi siempre a las mujeres si tienen dudas o quieren más información, el 43\% si les ha llegado la citación y el $95 \%$ les aconsejan que se realicen la mamografía.

Conclusiones: Los médicos de familia tienen buena opinión del programa de detección precoz de cáncer de mama, y creen en la efectividad de su consejo para mejorar la participación en él. Refieren falta de información, y en pocas ocasiones informan a la mujer sobre el programa.

Palabras clave: Medicina Familiar y Comunitaria. Cáncer de mama. Cribado. Conocimientos, Actitudes y Práctica en Salud.

Correspondencia:

Sofía Garrido Elustondo

Gerencia de Atención Primaria

Espronceda, 24. 28003 Madrid

Correo electrónico: sgarrido.gapm07@salud.madrid.org

ABSTRACT

\section{Opinion and Participation in the Regional Early Breast Cancer Detection Program in 2007 on the part of Family Physicians from a Health District in the Autonomous Community of Madrid, Spain}

Background: Mammogram screening is the most effective method for the early detection of breast cancer. The objective of this study is to evaluate the degree of knowledge, the opinion and the participation in the early breast cancer detection program on the part of the family physicians of the Autonomous Community of Madrid.

Methods: The population studied was comprised of family physicians from Madrid Health District Seven. An anonymous, self-administered questionnaire comprised of 30 questions grouped into physician's characteristics and opinion concerning the early breast cancer detection programs.

Results: A total of $46 \%$ of the physicians replied. A total of $94 \%$ of the physicians believed that it is their duty to inform their patients concerning preventive activities, including breast cancer screening, and $95 \%$ believed their advice to be useful for convincing women to have a mammogram. A total of $72 \%$ believed information to be lacking on this program. During the time when mammograms are being taken at their centres, $24 \%$ of the physicians surveyed always or almost always ask the women if they have any doubts or would like further information, $43 \%$ having set up appointments for them and 95\% advising them to have a mammogram taken.

Conclusions: The family physicians have a good opinion of the early breast cancer detection program and feel their advice to be effective for improving the participation in the program. They report lack of information and inform women about the program to only a small degree.

Key words: Family physicians. Breast neoplasm. Screening. Attitude. Health Knowledge, Attitudes, Practice. 


\section{INTRODUCCIÓN}

El cáncer de mama es la segunda causa específica de mortalidad en mujeres. En el año 2004, el cáncer de mama supuso el $12 \%$ de las defunciones en mujeres de 50 a 69 años, constituyendo así la principal causa de muerte en este grupo de edad $^{1}$. La realización de mamografías cada dos años en mujeres en este grupo es una medida eficaz para la detección temprana del cáncer de mama, lo que facilita un tratamiento precoz y menos agresivo. Sin embargo, para que esta medida sea efectiva en la reducción de la mortalidad es necesario asegurar una cobertura elevada de mujeres que se realizan la mamografía. En concreto, la reducción de la mortalidad por cáncer de mama en este grupo de edad puede llegar al 30\% tras 7 a 10 años de seguimiento, si se alcanzan coberturas de participación superiores al $70 \%{ }^{2}$.

Actualmente, todas las Comunidades Autónomas cuentan con programas de detección precoz de cáncer de mama que aconsejan a las mujeres de determinada edad de la población general a realizarse una mamografía con una periodicidad determinada $^{3}$. En Madrid, se desarrolla desde diciembre de 1998 el Programa de Detección Precoz de Cáncer de Mama de la Comunidad de Madrid (DEPRECAM). Este consiste en la realización cada dos años de una mamografía a todas las mujeres con edades comprendidas entre los 50 y $\operatorname{los} 69$ años. Las mujeres son citadas mediante carta personalizada para realizarse una mamografía en uno de los centros radiológicos concertados. Tras la exploración mamográfica con doble lectura posterior, se emite un informe radiológico y se envía, junto con las placas, a Atención Primaria.

No obstante la participación en el programa ha sido baja desde su inicio, siendo una de las comunidades autónomas con menor porcentaje de respuesta ${ }^{3}$. Con el objetivo de conocer las razones de esta baja participación, la Comunidad de Madrid (CM) llevó a cabo un estudio entre las mujeres que no acudieron a la cita. Este mostró que el motivo principal era la existencia de una mamografía previa en los dos años anteriores (58\%). En concreto, ésta estaba realizada en el $43,5 \%$ de los casos en un centro público, a pesar de que desde su implantación, todas las mamografías de cribado deberían realizarse a través de DEPRECAM.

El mejor instrumento para disminuir la mortalidad por cáncer de mama es conseguir una alta participación en programas de cribado poblacional ${ }^{4}$. Diferentes estudios han demostrado la importancia del papel del médico en los programas de detección precoz de cáncer de mama, ya que su consejo resulta efectivo para lograr una mayor participación de las mujeres en los mismos ${ }^{5-9}$. Por otro lado, para la adecuada planificación y gestión de todo servicio sanitario es conveniente conocer la opinión de todos los implicados en el mismo. Así, teniendo en cuenta el papel que juega el médico de familia en el programa, y en la línea de continuar investigando factores que puedan incrementar la participación de las mujeres, se planteó la realización de este estudio con el objetivo de valorar el grado de conocimiento, opinión y participación de los médicos de familia de la CM en el programa de detección precoz de cáncer de mama regional, así como su opinión sobre los programas de detección precoz de cáncer de mama en general.

\section{SUJETOS Y MÉTODOS}

Se realizó un estudio descriptivo transversal en el que la población de estudio fueron todos los médicos de familia del Área 7 de la CM $(\mathrm{N}=304)$. La razón de utilizar como población de estudio los médicos de 
esta área fue por un criterio de accesibilidad a dichos profesionales. Se trata de un área con una población adscrita de 550.000 habitantes, de ámbito exclusivamente urbano. El periodo de estudio comprendió la segunda quincena de junio y primera de julio de 2007.

La información se recogió mediante un cuestionario, anónimo y autoadministrado, elaborado ad hoc para el estudio. Se realizó un estudio piloto sobre una muestra de 20 médicos para valorar la comprensión del mismo, se introdujeron las modificaciones oportunas, y se elaboró el cuestionario definitivo. Este se envió por correo interno a cada médico. Se realizaron dos envíos, con un intervalo de dos semanas, para garantizar el mayor porcentaje de respuesta posible.

El cuestionario agrupa las preguntas en variables demográficas (edad, sexo), variables que recogen datos profesionales (situación laboral, titulación postgraduada, tipo de equipo de trabajo, presión asistencial media), preguntas en relación a los programas de detección precoz de cáncer (opinión sobre la efectividad de estos programas, y sobre qué institución debe gestionarlos), preguntas en relación a la detección precoz de cáncer de mama y preguntas en relación al DEPRECAM. En total contenía 30 preguntas: 27 cerradas y 3 abiertas.

El procesamiento y análisis de los datos se realizó mediante el programa SPSS v11, calculándose medias y desviaciones estándar (DE) en el caso de variables cuantitativas, y frecuencias absolutas y relativas para las cualitativas. Se realizó un análisis bivariante para valorar las posibles asociaciones entre algunas variables del estudio (ej. opinión sobre el programa) y las características de los médicos. Para realizar este análisis se utilizó el test de la ji cuadrado. Se aceptó un nivel de significación estadística del 5\% para todos los análisis.

\section{RESULTADOS}

Se recibió un total de 140 cuestionarios, lo que supone un porcentaje de respuesta del $46 \%$. En la tabla 1 se muestran las características demográficas y profesionales de los médicos de familia que contestaron la encuesta.

En respuesta a la opinión acerca de la efectividad de los programas de detección precoz de cáncer, el 1,4\% de los encuestados contestaron que muy alta, el 37,9 \% alta, el $42,1 \%$ aceptable, el $16,4 \%$ baja y el $0,7 \%$ muy baja. Se observó una relación significativa $(\mathrm{p}=0,035)$ con la edad del médico. Los médicos menores de 40 años, respondieron con mayor frecuencia que la efectividad era alta $(59,3 \%)$ o muy alta $(3,7 \%)$.

En cuanto a la opinión de los médicos sobre qué institución debería gestionar los programas de detección precoz de cáncer, el 51,1\% opinó que Atención Primaria, el

Tabla 1

Características de los médicos que contestaron la encuesta

\begin{tabular}{|l|c|}
\hline & $\mathbf{n}(\%)$ \\
\hline Sexo & \\
Hombre & $53(39,3)$ \\
Mujer & $82(60,7)$ \\
\hline Edad & \\
Media=45,4 años (DE=7,9) & \\
<40 años & $28(23,7)$ \\
$40-55$ años & $79(66,9)$ \\
$>55$ años & $11(7,9)$ \\
\hline Titulación & \\
MFyC vía MIR & $78(58,2)$ \\
MFyC no vía MIR & $31(23,1)$ \\
Médico General & $23(17,2)$ \\
Otra especialidad & $2(1,4)$ \\
\hline Situación laboral & \\
Plaza en propiedad & $93(68,4)$ \\
Interino & $38(27,9)$ \\
Suplente & $5(3,7)$ \\
\hline Equipo de Trabajo & \\
EAP & $128(94,1)$ \\
MT & $8(5,9)$ \\
\hline Presión asistencial & \\
$<36$ pac/día & $26(19,3)$ \\
36-45 pac/día & $57(42,2)$ \\
46-55 pac/día & $45(33,3)$ \\
$>55$ pac/día & $7(5,2)$ \\
\hline
\end{tabular}

EAP: Equipo de Atención Primaria. MT: Modelo Tradicional. 
Tabla 2

Respuestas de los médicos en relación a los programas de detección precoz de cáncer de mama

\begin{tabular}{|c|c|c|}
\hline & $\mathbf{n}$ & $(\%)$ \\
\hline \multicolumn{3}{|c|}{$\begin{array}{l}\text { La efectividad de la mamografía como método de detección pre- } \\
\text { coz de cáncer te parece }\end{array}$} \\
\hline $\begin{array}{l}\text { Muy baja } \\
\text { Baja } \\
\text { Aceptable } \\
\text { Alta } \\
\text { Muy alta }\end{array}$ & $\begin{array}{r}1 \\
7 \\
43 \\
71 \\
15\end{array}$ & $\begin{array}{r}(0,7) \\
(5,1) \\
(31,4) \\
(51,8) \\
(10,9)\end{array}$ \\
\hline \multicolumn{3}{|c|}{$\begin{array}{l}\text { Quien crees que debería captar a la población a la que va diri- } \\
\text { gida la mamografía de cribado }\end{array}$} \\
\hline $\begin{array}{l}\text { Programas institucionales } \\
\text { Consultas AP }\end{array}$ & $\begin{array}{l}90 \\
44\end{array}$ & $\begin{array}{l}(67,2) \\
(32,8)\end{array}$ \\
\hline \multicolumn{3}{|c|}{$\begin{array}{l}\text { Es función del médico de Atención Primaria informar a sus } \\
\text { pacientes acerca de las distintas medidas preventivas, incluida } \\
\text { el cribado del cáncer de mama }\end{array}$} \\
\hline $\begin{array}{l}\text { Muy en desacuerdo } \\
\text { Desacuerdo } \\
\text { Indeciso } \\
\text { De acuerdo } \\
\text { Muy de acuerdo }\end{array}$ & $\begin{array}{r}0 \\
4 \\
4 \\
81 \\
50\end{array}$ & $\begin{array}{r}(0,0) \\
(2,9) \\
(2,9) \\
(58,3) \\
(36,0)\end{array}$ \\
\hline \multicolumn{3}{|c|}{$\begin{array}{l}\text { Tu consejo como médico de Atención Primaria es útil para con- } \\
\text { vencer a tus pacientes de que se realicen una mamografía }\end{array}$} \\
\hline $\begin{array}{l}\text { Muy en desacuerdo } \\
\text { Desacuerdo } \\
\text { Indeciso } \\
\text { De acuerdo } \\
\text { Muy de acuerdo }\end{array}$ & $\begin{array}{r}1 \\
2 \\
4 \\
83 \\
49\end{array}$ & $\begin{array}{r}(0,7) \\
(1,4) \\
(2,9) \\
(59,7) \\
(35,3)\end{array}$ \\
\hline
\end{tabular}

10,9\% Atención Especializada, el 29,2\% Unidades específicas y el $8,8 \%$ otras instituciones. Se observó una relación estadísticamente significativa con la titulación del encuestado $(\mathrm{p}=0,019)$. Así, el 35,5\% de los especialistas no MIR opinó que estos programas se deberían gestionar desde Atención Primaria, frente al 57,3\% de los especialistas MIR y el $56,5 \%$ de los médicos generales.

En las tablas 2 y 3 se resumen las opiniones de los médicos en relación a los programas de detección precoz de cáncer de mama y al DEPRECAM, respectivamente. Destacan los siguientes resultados: el 94,3\% de los médicos están de acuerdo o muy de acuerdo en que es función del médico de familia informar a sus pacientes acerca de las actividades preventivas, incluidas el cribado de cáncer de mama; el $95 \%$ están de acuerdo o muy de acuerdo en que su consejo es útil para convencer a sus pacientes de que se rea-
Tabla 3

Respuestas de los médicos en relación de al Programa de Detección Precoz de Cáncer de mama de la Comunidad de Madrid (DEPRECAM)

\begin{tabular}{|l|l|l|}
\hline \multicolumn{2}{|c|}{$\mathbf{n}$} & $(\%)$ \\
\hline \multicolumn{2}{|c|}{ ¿Conocías la existencia de DEPRECAM? } & $\begin{array}{l}(95,7) \\
(4,3)\end{array}$ \\
\hline Sí & 134 & 6 \\
No & \multicolumn{1}{|c|}{} \\
\hline ¿Sabes desde qué institución se gestiona? & $(15,9)$ \\
\hline AECC & 22 & $(70,3)$ \\
SERMAS & 97 & $(13,8)$ \\
NS/NC & 19 &
\end{tabular}

¿Has recibido en los dos últimos años alguna información del Programa?

\begin{tabular}{|l|l|l|}
\hline $\begin{array}{l}\text { No } \\
\text { Sí }\end{array}$ & $\begin{array}{l}38 \\
101\end{array}$ & $\begin{array}{l}(27,3) \\
(72,7)\end{array}$ \\
\hline \multicolumn{2}{|c|}{ ¿Consideras que te falta información acerca del programa? } \\
\hline No & 39 & $(27,9)$ \\
Sí & 101 & $(72,1)$ \\
\hline En caso afirmativo, ¿De qué área? \\
\hline Fundamentos y Objetivo & 1 & $(0,7)$ \\
Interpretación de los datos & 13 & $(9,3)$ \\
Información a la mujer & 14 & $(10,0)$ \\
Conductas ante el resultado & 33 & $(23,6)$ \\
Circuito & 43 & $(30,7)$ \\
Todo el programa & 40 & $(28,6)$ \\
\hline
\end{tabular}

¿Te llega información específica de la fecha en la que se va a realizar la campaña?

\begin{tabular}{l|l|l} 
Nunca & 30 & $(21,7)$ \\
Algunas veces & 47 & $(34,1)$ \\
La mayoría de las veces & 45 & $(32,6)$ \\
Siempre & 16 & $(11,6)$
\end{tabular}

Siempre

$(21,7)$

$(32,6)$

$(11,6)$

El Médico de Atención Primaria debe ser quien informe del resultado

\begin{tabular}{|l|l|l}
\hline Muy en desacuerdo & 8 & $(5,9)$ \\
Desacuerdo & 31 & $(22,8)$ \\
Indeciso & 22 & $(16,2)$ \\
De acuerdo & 63 & $(46,3)$ \\
Muy de acuerdo & 12 & $(8,8)$ \\
\hline
\end{tabular}

El papel del médico de Atención Primaria en el programa, te parece

\begin{tabular}{l|l|l} 
Adecuado & 93 & $(71,0)$ \\
Insuficiente & 30 & $(22,9)$ \\
Excesivo, debería salir & 8 & $(6,1)$
\end{tabular}

¿Crees que el tiempo entre las mamografías que recomienda el Programa (cada 2 años) es el más adecuado?

\begin{tabular}{l|l|l}
\hline Sí & 97 & $(71,3)$ \\
No, debería ser anual & 25 & $(18,4)$ \\
NS/NC & 14 & $(10,3)$ \\
\hline
\end{tabular}

¿Crees que el grupo de edad hacia el que va dirigido el Programa es el más adecuado (50-69 años)?

\begin{tabular}{|c|c|c|}
\hline $\begin{array}{l}\text { Sí } \\
\text { No } \\
\text { Ilimitado } \\
\text { NS/NC }\end{array}$ & $\begin{array}{l}46 \\
79 \\
8 \\
5\end{array}$ & $\begin{array}{l}(33,3) \\
(57,2) \\
(5,8) \\
(3,6)\end{array}$ \\
\hline \multicolumn{3}{|c|}{$\begin{array}{l}\text { Durante el periodo de realización de mamografías en tu centro } \\
\text { ¿Te llega información específica de la fecha en la que se va a rea- } \\
\text { lizar la campaña? }\end{array}$} \\
\hline $\begin{array}{l}\text { Nunca } \\
\text { Algunas veces } \\
\text { La mayoría de las veces } \\
\text { Siempre }\end{array}$ & $\begin{array}{l}30 \\
47 \\
45 \\
16\end{array}$ & $\begin{array}{l}(21,7) \\
(34,1) \\
(32,6) \\
(11,6)\end{array}$ \\
\hline
\end{tabular}


Tabla 4

Frecuencia de realización de actividades relacionadas con el programa de detección precoz de cáncer de mama durante el periodo de realización de mamografías en el centro

\begin{tabular}{|c|c|c|c|c|}
\hline & Nunca & $\begin{array}{l}\text { Algunas } \\
\text { veces }\end{array}$ & $\begin{array}{l}\text { La mayoría } \\
\text { de las veces }\end{array}$ & Siempre \\
\hline & n $(\%)$ & n $(\%)$ & n $(\%)$ & n $(\%)$ \\
\hline $\begin{array}{l}\text { Preguntas a las mujeres que se encuentran en el grupo de edad si han } \\
\text { recibido la carta }\end{array}$ & $13(9,4)$ & $66(47,5)$ & $56(40,3)$ & $4(2,9)$ \\
\hline $\begin{array}{l}\text { Preguntas a las mujeres si tienen alguna duda en relación al programa o } \\
\text { si quieren saber algo más }\end{array}$ & $26(18,8)$ & $79(57,2)$ & $30(21,7)$ & $3(2,2)$ \\
\hline Aconsejas acudir cuando sean citadas a realizarse la mamografía & $3(2,2)$ & $4(2,9)$ & $29(20,9)$ & $103(74,1)$ \\
\hline
\end{tabular}

licen una mamografía; el $72,1 \%$ cree que le falta información acerca del programa. No se observaron diferencias en función de ninguna variable demográfica o profesional.

La tabla 4 resume la periodicidad con la que los médicos realizan determinadas actividades durante el periodo de realización de mamografías en su centro. Destaca que el $23,9 \%$ pregunta siempre o casi siempre a las mujeres si tienen alguna duda en relación al programa o quieren saber algo más; el $95 \%$ les aconsejan siempre o casi siempre que se realicen la mamografía; y el 43,2 les preguntan siempre o casi siempre si han recibido la carta.

En cuanto a la frecuencia con la que los médicos encuestados refieren derivar al servicio de ginecología para realizar mamografías de cribado, en el marco del Programa de Actividades Preventivas en Atención Primaria (PAPPS), no derivan nunca en el $41 \%$, algunas veces en un $40,8 \%$, la mayoría de las veces en $12,3 \%$ y siempre en $5,4 \%$.

En relación a la derivación al servicio de ginecología para realizar mamografías de cribado cuando la paciente lo solicita, los médicos refieren no hacerlo nunca en un $51 \%$, algunas veces en un $33,1 \%$, y la mayoría de las veces y siempre en el 9,9\% y $5,8 \%$ respectivamente.

La opinión general del programa de detección precoz de cáncer de mama de su
Comunidad (DEPRECAM) es buena o muy buena para el $80,2 \%$, indecisa para $19,8 \%$ y mala para el $3 \%$.

\section{DISCUSIÓN}

El porcentaje de respuesta ha sido de un $46 \%$. Esta cifra se considera aceptable, y está en la línea de otros trabajos publicados que utilizan la misma metodología.

Al comparar las variables sexo, edad, y trabajar en Equipo de Atención Primaria o Modelo Tradicional, no se han encontrado diferencias significativas entre los que contestaron al cuestionario y la población de estudio, por lo que podemos considerar nuestra muestra representativa de la población según estas variables. No obstante se debe tener en cuenta el sesgo de la no respuesta, ya que el hecho de no contestar el cuestionario puede traducir un menor interés en los programas de detección precoz de cáncer de mama, y por lo tanto una peor opinión y/o participación en los mismos.

Una de las principales limitaciones de este estudio puede ser la derivada de la utilización de un cuestionario no validado, pero no se encontró ninguno que cumpliese el objetivo del presente trabajo. No se han realizado estudios de validez y/o fiabilidad. Por otro lado, es la metodología que se suele utilizar en estudios con estos fines.

A pesar de que este estudio se enmarca en un área de Madrid concreta, no se han 
encontrado razones que indiquen que existen diferencias entre los médicos de familia que trabajan en esta área y el resto de los médicos de familia de la $\mathrm{CM}$, por lo que los resultados se podrían generalizar al resto de la Comunidad.

Este estudio muestra que el $39 \%$ de los médicos considera que la efectividad de los programas de detección precoz de cáncer es alta o muy alta. Este discreto porcentaje puede ser debido a una falta de formación o conocimiento sobre los mismos, que se observa también en otros estudios ${ }^{10-11}$. De hecho, los médicos más jóvenes, a los que se les presupone una mayor formación en este campo, respondieron con mayor frecuencia que la efectividad era alta o muy alta.

Frente a este dato, un mayor porcentaje de médicos (63\%), consideran alta o muy alta la efectividad de los programas de detección precoz de cáncer de mama mediante la realización de una mamografía de cribado. Quizás sea debido a un mayor conocimiento de los mismos debido a la amplia implementación de los programas de detección precoz de cáncer de mama en nuestro medio.

En relación al papel del médico de familia en los programas de diagnóstico precoz de cáncer, una amplia mayoría estaba de acuerdo con que entre sus funciones se incluía el informar a sus pacientes de las distintas medidas preventivas. Este dato coincide con otros estudios en los que los médicos encuestados consideraban que deben asumir un papel activo en los programas poblacionales de detección precoz de cáncer ${ }^{11-12}$. Además, en un estudio que analizaba las creencias y actitudes hacia el cáncer de mama y la mamografía asociadas con la participación en un programa de cribado mamográfico, se evidenció la importancia de la información recibida por su médico en la asistencia o no a los programas de prevención ${ }^{13}$.
Llama la atención la falta de información del programa DEPRECAM por parte de los médicos de familia. A pesar de que los médicos encuestados dicen conocer el programa, el $30 \%$ desconocía quien lo gestiona y el $72 \%$ considera que le falta información, especialmente respecto al circuito. Además el $27 \%$ refiere no haber recibido información acerca del programa en los dos últimos años. Este hecho resulta llamativo, debido a las repetidas ocasiones en que, dentro de las actividades del programa, se envía información a los médicos. Se observa por tanto disparidad entre la información emitida y la recepción y/o percepción de estar informados de nuestros profesionales. En cuanto al acuerdo con aspectos específicos del programa, la mayoría de los médicos $(71 \%)$ consideran el tiempo recomendado entre las mamografías, cada dos años, adecuado. El porcentaje que muestran su acuerdo con el grupo de edad a quien va dirigido el programa, 50 a 69 años, es menor (33\%). Esto puede estar relacionado con la falta de consenso científico existente en la actualidad en la recomendación de cribado en mujeres menores de 50 años ${ }^{1}$.

Otro hallazgo en nuestro estudio es que el $95 \%$ de los médicos de familia considera que su consejo resulta útil para convencer a las pacientes para que se realicen una mamografía. De hecho, durante el periodo de realización de mamografías en el centro, el $74 \%$ de los médicos refieren aconsejar siempre acudir a la cita. Este dato es importante, ya que diferentes estudios concluyen que el consejo médico aumenta en gran medida la realización de mamografía por parte de las mujeres en el grupo de edad recomendada ${ }^{4-8}$.

Sin embargo observamos poca implicación de los médicos en otras actividades relacionadas con el programa. Así, el 57\% de los encuestados no pregunta con asiduidad a sus pacientes si han recibido la carta de citación durante el periodo de 
realización de mamografías en su centro, y sólo el $23 \%$ pregunta siempre o la mayoría de las veces a sus pacientes si tienen dudas respecto al programa o quieren mayor información durante ese periodo. Las creencias y los conocimientos de la mujer sobre cáncer de mama y sus técnicas de detección temprana tienen una clara influencia en la participación de las mujeres en los programas de cribado poblacional $^{13-}{ }^{16}$. El médico de familia puede actuar sobre estas creencias y temores negativos, trasmitiendo una información tranquilizadora y favoreciendo en definitiva la participación de las mujeres en estos programas.

Destaca el porcentaje de derivaciones al servicio de ginecología para la realización de una mamografía de cribado que refieren los médicos, teniendo en cuenta que desde la implementación del Programa en su Comunidad, todas las mamografías de cribado deberían realizarse a través del mismo. Este dato coincide con los resultados del estudio que investigaba las causas por las que las mujeres no acudían a la cita, siendo la principal la existencia de una mamografía previa en los dos años anteriores, realizada además en el 43,5\% de los casos en un centro público. Este hecho podría responder a una falta de información sobre el programa.

Analizando los trabajos publicados, se observa que en nuestro medio se han estudiado factores que predisponen a las mujeres a la asistencia a los programas de cribado mamográfico y causas de no asistencia a dichos programas, pero no se ha encontrado ningún estudio que analice la opinión, y actitudes de los médicos de familia en relación con estos programas. Sin embargo, éstos se encuentran en una posición muy favorable para ayudar a las mujeres a realizarse la mamografía debido a la accesibilidad, la frecuentación de los servicios de las mujeres a esa edad y la influencia que pueden ejercer sobre sus decisiones. Este estudio es por lo tanto útil para determinar en qué aspectos se puede incidir para mejorar la participación de las mujeres.

Como conclusión, nos encontramos con unos médicos con una buena opinión del programa, y que creen en la efectividad de su participación en el mismo. Sin embargo se implican poco en algunas actividades concretas del programa y refieren falta de información. Proponemos mejorar y mantener activa la información acerca del programa, tanto del circuito, como de los resultados del mismo y protocolizar y fomentar determinadas actividades por parte de nuestros médicos dirigidos a transmitir a las mujeres una información tranquilizadora sobre los resultados más probables de la prueba, incidir en la utilidad del cribado mamográfico y especificar cómo se realiza la prueba y en qué consiste.

Sería interesante asimismo repetir este estudio tras haber implementado las nuevas medidas recomendadas y valorar así el impacto que estas hayan podido tener en la participación y conocimiento de los médicos de atención primaria respecto a los programas de cribado de cáncer de mama.

\section{BIBLIOGRAFÍA}

1. Instituto Nacional de Estadística. Defunciones según causa de muerte. (Citado el 29 de enero de 2007). Disponible en: http://www.ine.es/prensa

2. Agencia de Evaluación de Tecnologías Sanitarias (AETS). Instituto de Salud Carlos III- Ministerio de Sanidad y Consumo. Cribado Poblacional de Cáncer de Mama mediante Mamografía. Madrid: AETS - Instituto de Salud Carlos III; 1995.

3. Agencia de Evaluación de Tecnologías Sanitarias (AETS). Instituto de Salud Carlos III- Ministerio de Sanidad y Consumo. Uso de la mamografía y de la citología de Papanicolaou para la detección precoz de cáncer de mama y de cérvix uterino en España. Madrid: AETS - Instituto de Salud Carlos III; 2002. 
4. Gøtzsche PC, Nielsen M. Cribaje (screening) con mamografía para el cáncer de mama (Revisión Cochrane traducida). En: La Biblioteca Cochrane Plus, 2007 Número 2. Oxford: Update Software Ltd. Disponible en: http://www.update-software.com.

5. Giveon Shmuel, Kahan E. Patient adherente to family practitioners' recommendations for breast cancer screening: a historical cohort study. Fam Pract. 2000; 17: 42-5.

6. Chaudhry R et al. Web-Based proactive system to improve breast cancer screening. Arch Intern Med 2007; 167: 606-11.

7. Hawley ST, Earp JA, O*Malley M, Ricketts TC. The role of physician recommendation in women's mammography use: is it a 2 stage process? Med Care 2000; 38: 392-403.

8. Giorgi D, Gionardo L, Senore C, Merlino G, Negri R, Cancian M et al. General practitioner and mamographic screening uptake: influence of different modalities of general practitioner participation. Working Group. Tumori 2000; 86: 124-9.

9. Meissner HI, Breen N, Taubman ML, Vernon SW, Graubard BI. Which woman aren't getting mammograms and why? Cancer Causes Control. 2007; 18: 61-70.

10. Santora LM, Mahoney MC, Lawvere S, Englert JJ, Symons AB, Mirand AL. Breast cancer screening beliefs by practice location. BMC Public Health. 2003 Feb 4;3:9. Disponible: http://www.biomedcentral.com/1471-2458/3/9.

11. Escher M, Sappino A. Primary care physicians' knowledge, and attitudes towards genetic testing for breast-ovarian cancer predisposition. Ann. Oncol. 2000;11: 1131-5.

12. Pichert G, Dietrich D, Moosmann P, Zwahlen M, Aarón R, Appino A. Swiss primary care physicians'knowledge, attitudes and perception towards genetic testing for hereditary breast cancer. Fam Cancer. 2003; 2: 153-8.

13. Andreu Y, Galdón MJ, Durá E; Carretero S, Tulles J. Edad, creencias de salud y asistencia a un programa de cribado mamográfico en la comunidad valenciana. Rev Esp Salud Publica. 2004; 78: 65-82.

14. Alcaraz M, Llucu A, Miranda J, Pereiro I, Salas MD. Estudio de la no participación en el programa de prevención de cáncer de mama en la ciudad de Valencia. Gac Sanit 2002; 16: 230-5.

15. Luengo S, Azcona B, Lázaro P, Madero R. Programas de detección precoz del cáncer de mama y acceso a la mamografía en España. Med Clin (Barc) 1997; 108: 761-5.

16. George S.A: Barriers to breast cancer screening: an integrative review. Health Care Women Int. 2000; 21: 43-65. 